\title{
Influence of parental eating disorder on children
}

\author{
H. Woolley, R. Wheatcroft \& A. Stein
}

It is now well established that children of parents who have psychiatric disorders are at risk of developing disturbances themselves (Rutter, 1989; Garmezy \& Masten, 1994). Of particular concern is that these disturbances may persist well after remission of the parental disorder. Eating disorders occur commonly among women of child-bearing age (Szmukler, 1985; Fairburn \& Beglin, 1990) and the prevalence seems to be rising (Treasure et al, 1996). Estimates indicate that at least $2 \%$ of women aged 16-40 suffer from bulimia nervosa or anorexia nervosa, and up to $4 \%$ when other eating disorders are considered (Fairburn \& Beglin, 1990; Hoek, 1993). It is therefore surprising that the potential implications for their offspring have received so little attention until recently. Knowledge of whether these children are at risk and the mechanisms underlying any transmission of disturbance is important so that appropriate treatment can be instituted and preventive strategies put in place.

There are a number of reasons why an eating disorder may potentially interfere with the quality of parenting. First, the core symptoms of eating disorders are extremely pervasive and potentially disruptive of daily activities and sensitive parenting. Particularly debilitating are the preoccupations with body shape, weight and food, which may draw parental attention away from the needs of their child. The behaviours which characterise eating disorders and which may disrupt harmonious parent-child interaction include episodes of binge eating, and the extreme behaviours to compensate for over-eating, such as vomiting, the use of purgatives and excessive exercise. Second, people with eating disorders have difficulties in their interpersonal relationships (Humphrey, 1989), and it is of concern that these difficulties may extend to their relationships with their children. Furthermore, particular stages of development may render children especially susceptible to the influence of parental eating disorders.

\section{Infancy}

This may be a vulnerable time given that feeding and mealtimes take up a significant part of the day during the first months and years of life, and are one of the most important times for close communication between parents and children. It is through feeding that children first experience some of their new developmental capacities, particularly those of self-gratification and autonomy. At these times children require particularly sensitive and facilitative parenting to promote the child's exploratory behaviour. However, the attitudes, preoccupations and behaviours that people with eating disorders manifest may interfere with their ability to prepare meals, to sit patiently feeding their infants, and to respond appropriately to hunger and satiety cues. Although eating disorder symptomatology may diminish during pregnancy, recent research has shown that the disturbance rises in the postpartum period to above pre-pregnancy levels, with mothers trying to regain their prepregnancy body shape and weight in the context of the significant disruption of their own mealtimes and sleep routines while feeding and caring for their baby (Fairburn et al, 1993; Stein \& Fairburn, 1996).

\footnotetext{
Helen Woolley and Becky Wheatcroft work at the Royal Free Hospital School of Medicine in a longitudinal study of children's feeding, growth and development in the context of maternal eating disorders, focusing upon detailed observations of parent-child interactions through the early years. Alan Stein is the Leopold Muller Professor of Child and Family Mental Health at the Royal Free Hospital School of Medicine (Rowland Hill Street, London NW3 2PF). He has a long-standing interest in the intergenerational transmission of psychological disturbance, especially of eating disorders. He also has an interest in family factors in feeding disturbances in young children. Their research is funded by the Wellcome Trust (grant no. 035035).
} 


\section{Adolescence}

Adolescence is a time when children become more aware of societal pressures and develop increasing interest in body shape and attractiveness. A recent study from the USA indicated that weight control was the single most important concern in an epidemiological study of health concerns among a large sample of Californian teenagers (Evans $\mathrm{et} \mathrm{al,}$ 1995). Parents' attitudes towards their own body shape and weight, and their own behaviour with respect to eating, may affect their adolescent children in two ways. First, the children may model themselves on their parents; and second, the parents may influence their adolescent children directly by attitudes towards their children's weight, shape and eating habits.

\section{Evidence from research}

Most studies on the development of children of parents with eating disorders have consisted of clinical case reports and case series in which concerns are raised about the risk of adverse sequelae for the children. Given the nature of these reports it is not easy to evaluate their significance. Studies have concentrated on mothers with eating disorders with little reference to fathers. Two of these reports found evidence that the mother's disorder impinged directly on the child. Lacey \& Smith (1987) found that some women with bulimia nervosa were concerned that their babies were overweight and had tried to slim them down, while Stein \& Fairburn (1989) found that some mothers with bulimia nervosa were excessively worried about their children's shape and size and also had significant difficulties with feeding their children. Others have also found that the mothers' eating disorders interfered with the general parenting of children (Fahy \& Treasure, 1989; Woodside \& Shekter-Wolfson, 1990; Timimi \& Robinson, 1996). Fahy \& Treasure (1989) described the conflicting demands of child rearing for parents coping with severe bulimic episodes while having to feed their young children, when the very presence of food may stimulate a binge. More recently, Hodes et al (1997) investigated the extent of psychiatric disorder in 26 children of 13 mothers with eating disorders, and found that $50 \%$ of the children (age range 9 months to 25 years) had psychiatric disorders, including severe conditions such as anorexia nervosa and obsessive-compulsive disorder.
There is also evidence that the physical growth of children of mothers with eating disorders might be adversely affected. Brinch et al (1988) followedup a group of women with a history of anorexia nervosa, who had subsequently had children, and reported that $17 \%$ of these children had failed to thrive in the year following birth. This finding should be regarded with some caution because these figures were based largely on retrospective maternal reports of child development and on a broad definition of failure to thrive. Van WezelMeijler \& Wit (1989) reported that seven children from three mothers with eating disorders presented with features of growth retardation, which the authors believe was due to under-nourishment of the child. Furthermore, Hodes et al (1997) found that one-third of children of mothers in their case series were significantly lighter or smaller than expected for their age.

There has been one published controlled study that employed direct observation of the one-yearold children of mothers with eating disorders (Stein et al, 1994). Mothers and infants were observed during both mealtimes and play in the home. The main findings were that, when compared with controls, the index mothers were more intrusive with their infants during both mealtimes and play, and they expressed more negative emotion (critical and derogatory remarks) during mealtimes but not during play. The most common precipitant of such expressed negative emotion was the mothers' concern that their infants were making a 'mess'. There were, however, no differences between the groups in the extent of their positive expressed emotion towards their infants. There was considerably more conflict between index infants and their mothers during mealtimes, with the index mothers showing much more reluctance to allow their infants to attempt self-feeding, compared with the control mothers. Mess avoidance and the need to keep control of food intake seemed particularly important to these mothers. Furthermore, the index infants weighed less than the control infants, and infant weight was found to be independently and inversely related to the amount of conflict during mealtimes. It should be emphasised that while a significant proportion of mothers and infants were having problems, this was not invariable. Some mothers were coping well with their babies, who were growing and developing healthily (Stein et al, 1994).

In order to establish whether growth faltering among children of mothers with eating disorders was specific, this study was extended. The infants of mothers with eating disorders were compared with infants of mothers with postnatal depression and a large comparison group (Stein et al, 1996). 
It was found that infants of mothers with eating disorders were smaller, both in terms of weight and length for age, than either the normal comparison group or the infants of mothers with postnatal depression; and the latter two groups did not differ from each other (Stein et al, 1996). This indicated that the growth faltering was specific to the infants of mothers with eating disorders.

The same two groups were used to examine whether or not there were more direct influences than conflict on growth disturbance. Two features of maternal psychopathology might lead the mothers to limit their children's food intake and potentially bring about growth disturbance in the children. First, people with eating disorders tend to misjudge their own body shapes, by perceiving themselves to be fatter than they actually are. Second, they have a strong desire to be thin. These mothers' misjudgements about their own body shape could be extended to their evaluation of their infants' body shape. If they desire their infants to be thin, they may limit their children's food intake. However, empirical examination revealed that, compared with the mothers with depression and the comparison group, the mothers from the eating disorder group did not prefer thinner babies, and they did not misperceive their children's size. On the contrary, the mothers with eating disorders were highly sensitive to their children's shape and, compared with the other two groups, were significantly more accurate at judging their children's size (Stein et al, 1996).

The strength and specificity of the association between maternal eating disorder psychopathology and child disturbance was confirmed by a study using a different strategy, namely, one which used the child's disturbance as a starting point (Stein et al, 1995). The mothers of a consecutive series of young children referred to child psychiatric clinics with ICD-10 (World Health Organization, 1992) feeding disorders were compared with a matched group of children with behavioural disorders and a large unselected control group. ICD-10 criteria require that a feeding disorder in infancy or childhood "generally involves refusal of food and extreme faddiness". It was found that compared with the other two groups, only the children with feeding disorders had mothers with significantly disturbed eating habits and attitudes. The finding that children's feeding disorders were specifically linked to the mothers' eating habits and attitudes suggests that maternal eating psychopathology may play a significant role in the development of children's feeding disorders (Stein et al, 1995). There are a number of different types and variations in the presentation of eating and feeding disorders in children. These have been well reviewed by Bryant-Waugh \& Kaminski (1993; see Box 1) and Bryant-Waugh \& Lask (1995). Two disorders are particularly worthy of mention. The term 'food avoidance emotional disorder' (FAED), first used by Higgs et al (1989), describes children who have a disorder of emotions in which food avoidance plays a prominent part, but who do not meet diagnostic criteria for anorexia nervosa. 'Selective eating' is a term used for those children who have for many years had a very restricted range of foods. Typically they eat only three or four different foods, usually carbohydrate based, such as biscuits, potatoes and bread. Initially they present as having food fads, but unlike most faddy children their range of foods does not increase. Often they present to a paediatrician or child psychiatrist at age 8-12 years because of parental concern about the child's health. Such children tend to be physically healthy, and their weight and height is within the normal range (Bryant-Waugh \& Kaminski, 1993). However, there has been no systematic research to examine the precise relationship between the different eating or feeding difficulties in childhood and parental eating psychopathology, and whether certain types of childhood difficulty are particularly related to parental eating disturbance.

A few studies have looked at the potential relationship between eating disorders in older children and those of their mothers, the aim being to consider whether mothers' attitudes and behaviours regarding food are a factor in determining whether or not their daughters would have disordered eating. This was the focus of a study by Pike \& Rodin (1991) in America, who used self-report measures in groups of middle-class mothers and adolescent daughters. They compared mothers of daughters with disordered eating (assessed by the Eating Disorder Inventory) with control subjects. They found that mothers whose daughters' eating was disordered had more eating problems themselves. Mothers of girls with disordered eating also thought that their daughters should lose more weight than did the mothers of girls who had no

Box 1. Types of eating and feeding disorders in children (Bryant-Waugh \& Kaminski, 1993)

Anorexia nervosa

Food avoidance emotional disorder

Food refusal

Pervasive refusal

Selective eating

Bulimia nervosa

Appetite loss secondary to depression 
such disorder, and the former also thought their daughters were less attractive than the girls judged themselves. The study by Attie \& BrooksGunn (1989), however, did not support these findings. A third, very small, UK study of 10 -yearold girls and their mothers, found that mothers' and daughters' dietary restraint scores were correlated, but that their eating attitudes (as measured by the Eating Attitudes Test) were not (Hill et al, 1990). These issues, therefore, remain largely unresolved. Research has yet to be carried out to establish any causal link between disordered eating behaviours in mothers and their children.

It should again be emphasised that the children of parents with eating disorders are not invariably adversely affected. Some parents manage well and their children develop without apparent problems. Thus, the importance of assessing each family carefully cannot be overemphasised.

\section{Mechanisms of transmission}

From the relatively limited number of studies conducted to date, it is possible to describe five broad mechanisms by which parents with eating disorders may influence child rearing and their children's development (see Box 2). First, parents' extreme attitudes to eating, body shape and weight, may have direct effects on the child. For example, parents' fear of fatness may cause them to underfeed their children; and their over-concern with shape, weight and food intake, may also lead them into conflict with their children during mealtimes and into being very critical of their adolescent children's eating habits, body shape and appearance. Second, a parent's eating disorder may interfere with general parenting functioning. For example,

Box 2. Possible mechanisms by which parents with eating disorders may influence child rearing and their children's development

Direct influence - parents' extreme attitudes to eating, body shape and weight

Indirect influence - preoccupations interfere with sensitivity and responsiveness to child needs

Parents' disturbed eating behaviour act as role model

Influence of associated family discord

Genetic parents with eating disorders tend to be preoccupied with food, body shape and weight, and these preoccupations may impair their concentration in such a way as to interfere with their sensitivity and responsiveness to their children's needs. Third, parents' disturbed eating behaviour may act as a poor role model for their children. Fourth, parents' eating disorder may be associated with discordant marital and family relationships, which have been shown to have their own adverse effects on child development (Rutter \& Quinton, 1984). Fifth, it is always important to consider genetic effects, and while relatively little has been done to illuminate how parental eating disorders may influence child rearing and the child's general development through genetic factors, there is now evidence that there is a genetic component to eating disorders (Holland et al, 1984; Kendler et al, 1991).

\section{Clinical Implications}

Given the relative paucity of the research, only tentative guidelines can be proposed, but on the basis of our own research and clinical experience, we have developed a clinical approach to families with young children where a parent has an eating disorder. Most of our comments are confined to helping families with young children, although a number of the issues are relevant to older children as well. These guidelines are not only intended for clinicians working with young families, but also for those working with parents with eating disorders, who are well-placed to identify any difficulties which occur in parent-child interaction or in child development (Berg \& Hodes, 1997), and either to deal with these themselves or refer on as necessary. It is also essential that the parental eating disorder is itself treated.

\section{Assessment and treatment as an integral process (Box 3)}

\section{Child's growth and parent-child interaction}

At the outset, it is important that the quality of the interactions between parents with eating disorders and their children be carefully and clinically assessed, as should the children's growth and nutritional status.

\section{Observe mealtime at home}

Observing mealtimes in the home, as sensitively and unintrusively as possible, is a particularly 
useful part of that assessment since the clinician can observe the natural, everyday, mealtime parentchild interaction. This is important because much communication between parents and infants occurs around mealtimes when potential conflicts may arise. Differences and disputes, over events such as an infant's attempt to self-feed, are a normal part of mealtime experience. Negotiation of these differences signals to the child that such disputes are normal events and are resolvable. Some parents, however, overburdened by their own eating difficulties, get locked into disputes with their infants, and extensive conflictual interaction may become the norm, causing great distress to both parent and child. It is important, therefore, to help parents to identify their own 'flashpoints' (or precursors to conflict) and work out some simple defusion techniques.

\section{Help parents to recognise infant cues}

A parent's ability to recognise, acknowledge and respond to the infant's signals and to understand age-appropriate needs is a key factor for managing conflict. Particular help may be needed in recognising an infant's hunger and satiety cues. As Chatoor et al (1988) point out, infants may have difficulty learning about their own somatic and emotional states if parents constantly misread infant cues and reflect back those misperceptions.

\section{Identify those parental concerns that interfere with the recognition of cues}

Sometimes the parents' own concerns, stemming from extreme attitudes to body shape, weight and food, colour the way they perceive and understand their child's cues. For instance, a parent may feed an infant very rapidly, wishing to get the feed over quickly for fear that the very presence of food may precipitate a binge, thus missing or ignoring the fact that the infant is not ready for the next food offer (he or she still has a mouthful), and setting the scene for a struggle over persistent food offers and ultimately food refusal. Sympathetic understanding that avoids blame is crucial here to help the parent acknowledge their own concern and read the situation from the infant's viewpoint.

Disputes around persistent food refusal may arise for a variety of reasons besides mispacing of food offers. For instance, solid food may be persistently offered only after a full milk feed has been given, so that it is lack of hunger that leads to refusal rather than any disinterest in or dislike of the solid food. It is also worth suggesting varying the range and amount of food offered.

\section{Help manage triggers of mealtime disputes}

Dealing with mess is a common difficulty for many parents with an eating disorder. Extreme concern about mess and the wish to maintain control can easily lead to a refusal to allow the infant to selffeed and experiment with food. On an emotional level such parents need help in dealing with their strong aversion to perceived mess while accepting that some mess may be inevitable as an infant learns to self-feed. Small practical suggestions can be useful, such as using a waterproof tablecloth under the high chair, using two spoons (one for parent, one for infant) and possibly two feeding dishes to allow the infant to experiment with smaller amounts of food. With the need to avoid mess at all costs some parents refuse to allow the infant to touch food with their hands (finger feed) and may have unrealistic expectations about the infant's deftness with utensils. Subsequent negative derogatory comments directed at the infant may undermine and deter their efforts, triggering a further conflictual cycle. Separating out the parental concern about mess from the child's developmental needs is important here, so that the parent can reframe their perception of the infant's self-feeding attempts, and even feel a pride in the infant's developing skills and autonomy.

\section{Observe regulatory style of parent}

The style of the caretaker's regulatory demands and the nature and consistency of prompts to eat, whether highly directive or facilitative, has been the focus of study (Klesges et al, 1983; Chess \& Hassibi, 1986); the latter arguing that "the majority of eating disturbances in early childhood are caused by the child's reaction to the regulatory demands of his caretakers". Early feeding battles may begin, they suggest, with the feeding preferences of the child and the regulatory demands of a rigid parent. In our own study (Stein et al, 1994), a directive controlling style in relation to infant feeding was not in itself related to conflict. If, however, the maternal demands and directives cut across the infant's own initiatives, then feeding difficulties were more likely to arise. The issue here concerned the extent of the mother's sensitivity towards her infant, rather than the nature of her directives (commands versus prompts and encouragements). In assessing interaction it is therefore useful to note the parent who directs the infant in such a way as to assert absolute control without reference to the infant's signals or needs. Such control is a one-way process excluding the infant's perspective. This contrasts with the directive style that nevertheless encompasses two-way 
Box 3. Parents with eating disorder feeding young children: guidelines for mealtime help

Avoid blaming parents

Observe a mealtime in the home (if possible) to identify interactional style, and both positive and negative engagement

Highlight and support child's exploratory behaviour

Elicit the main parental mealtime concerns (e.g. mess) and work on managing these

Help parents to identify mealtime flashpoints and to manage the associated anxiety

Help parent recognise infant signals (e.g. hunger, satiety, readiness for more food) and pace food offers accordingly

Avoid starting meals when child is already full (e.g. after a full bottle of milk)

Help the parent to recognise child as separate person with their own needs

It may be helpful to provide a wide variety of food in smaller portions

Widen the focus of mealtime 'talk' to defuse tension around food

Recruit support during mealtimes from the partner or another person (e.g. a parent)

responses and gives affectionate containment. A useful question here is whether the parent can accommodate or tolerate the infant's perceived preferences, even when they contravene their own beliefs about food.

\section{Help with limit-setting and anger management}

While most parents may have particular family 'rules' about eating manners, some may need help in setting realistic and age-appropriate rules, in particular setting limits without mocking or getting angry with the child. Recognition of how the parent feels when rules are broken, and how to contain and manage those feelings, may be crucial in avoiding the escalation of dispute into full conflict once feeding flashpoints occur. For some parents even a minor breach of a rule represents a threatening loss of control, and simple low-key ploys may help them to keep a sense of control without heavyhanded intrusion.

\section{Help widen the focus of mealtimes}

Where older children are involved, help might take the form of working with parents to lessen the attention and criticism directed at their children's body weight and shape, where this is a problem. It is especially important with this age group that the parents should be helped to widen the focus of their interaction with their children during mealtimes, encouraging discussion of the day's events, trying to focus on areas of mutual enjoyment. This becomes particularly important for those families where excessive concerns about eating, body weight and shape have become major vehicles for family communication and mutual valuation (Kalucy et al, 1977; Katzet al, 1985).

\section{Consider drawing in help of partner/supporter}

Struggling to feed a child can be a very lonely experience, especially if parent and child have got into intractable conflict. The sensitive involvement of a spouse or partner (being careful not to undermine the parent) may be supportive in sharing or even taking over the feeding at some mealtimes. The presence of another adult can also help to diffuse the intense conflict, and the partners can work together and learn to identify the child's feeding patterns, cues and needs.

\section{References}

Attie, I. \& Brooks-Gunn, J. (1989) Development of eating problems in adolescent girls: a longitudinal study. Developmental Psychology, 25, 70-79.

Berg, B. \& Hodes, M. (1997) Adult psychiatrists' knowledge of children whose mothers have eating disorders. European Eating Disorders Review, 5, 25-32.

Brinch, M., Isager, T. \& Tolstrup, K. (1988) Anorexia nervosa and motherhood: reproduction pattern and mothering behaviour of 50 women. Acta Psychiatrica Scandinavica, 77, 611-617.

Bryant-Waugh, R. \& Kaminski, Z. (1993) Eating disorders in children. In Childhood Onset Anorexia and Related Eating Disorders (eds B. Lask \& R. Bryant-Waugh), pp. 17-29. Hove: Lawrence Erlbaum.

— \& Lask, B. (1995) Annotation: eating disorders in children. Journal of Child Psychology and Psychiatry, 36, 191-202.

Chatoor, I., Egan, J., Getson, P., et al (1988) Mother-infant interactions in infantile anorexia nervosa. Journal of the American Academy of Child and Adolescent Psychiatry, 27, 535-540.

Chess, S. \& Hassibi, M. (1986) Principles and Practice of Child Psychiatry (2nd edn), p. 221. New York: Plenum.

Evans N., Gilpin E., Farkas A. J., et al (1995) Adolescents' perceptions of their peers' health norms. American Journal of Public Health, 85, $1064-1069$.

Fahy, T. \& Treasure, T. (1989) Children of mothers with bulimia nervosa. British Medical Journal, 299, 1031.

Fairburn, C. G. \& Beglin, S. (1990) Studies of the epidemiology of bulimia nervosa. American Journal of Psychiatry, 147, 401-408.

-, Jones, R. \& Stein, A. (1993) Eating habits and attitudes during pregnancy. Psychosomatic Medicine, 54, 665-672.

Garmezy, N. \& Masten, A. (1994) Chronic adversities. In Child and Adolescent Psychiatry (eds M. Rutter \& L. Hersov), pp. 191-208. Oxford: Blackwell.

Higgs, J., Goodyer, I. \& Birch, J. (1989) Anorexia nervosa and food avoidance emotional disorder. Archives of Disease in Childhood, 64, 346-351. 
Hill, A. J. ,Weaver, C. \& Blundell, J. E. (1990) Dieting concerns of 10 year old girls and their mothers. British Journal of Clinical Psychology, 29, 346-348.

Hodes, M., Timimi, S. \& Robinson, P. (1997) Children of mothers with eating disorders: a preliminary study. European Eating Disorders Review, 5, 11-24.

Hoek, H. W. (1993). Review of the epidemiological studies of eating disorders. International Review of Psychiatry, 15, 346-348.

Holland, A. J., Hall, A., Murray, R., et al (1984) Anorexia nervosa: a study of 34 twin pairs and one set of triplets. British Journal of Psychiatry, 145, 414-419.

Humphrey, L. L. (1989) Observed family interactions among subtypes of eating disorders using structural analysis of social behaviour. Journal of Consulting and Clinical Psychology, 57, 206-214.

Kalucy, R. S., Crisp, A. H. \& Harding, B. (1977) A study of 56 families with anorexia nervosa. British Journal of Medical Psychology, 50, 381-395.

Katz, R. L., Mazer, C. \& Litt, I. F. (1985) Anorexia nervosa by proxy. Journal of Pediatrics, 107, 247-248.

Kendler, K. S., MacLean, C., Neale, M., et al (1991) The genetic epidemiology of bulimia nervosa. American Journal of Psychiatry, 148, 1627-1637.

Klesges, R.C., Coates, T., Brown, G., et al (1983) Parental influences on children's eating behavior and relative weight. Journal of Applied Behavior Analysis, 16, 371-378.

Lacey, J. H. \& Smith, G. (1987) Bulimia nervosa: the impact of pregnancy on mother and baby. British Journal of Psychiatry, 150, 777-781.

Pike, K. M. \& Rodin, J. (1991) Mothers, daughters, and disordered eating. Jourmal of Abnormal Psychology, 100, 198-204.

Rutter, M. (1989) Psychiatric disorder in parents as a risk factor for children. In Prevention of Mental Disorder, Alcohol and Other Drug Use in Children and Adolescents (eds D. Schaffer, I. Phillips \& N. B. Enger), pp. 157-189. Rockville, MD: Office for Substance Abuse.

— \& Quinton, D. (1984) Parental psychiatric disorder: effects on children. Psychological Medicine, 14, 853-880.

Stein, A. \& Fairburn, C. G. (1989) Children of mothers with bulimia nervosa. British Medical Journal, 299, 777-778.

- Woolley, H., Cooper, S. D., et al (1994) An observational study of mothers with eating disorders and their infants. Journal of Child Psychology and Psychiatry, 35, 733-748.

-, Stein, J., Walters, E. A., et al (1995) Eating habits and attitudes among mothers of children with feeding disorders. British Medical Journal, 310, 228.

- \& Fairburn, C. G. (1996) Eating habits and attitudes to body shape and weight during the postnatal period. Psychosomatic Medicine, 58, 321-325.

- Murray, L., Cooper, P., et al (1996) Infant growth in the context of maternal eating disorders and maternal depression: a comparative study. Psychological Medicine, 26, 569-574.

Szmukler, G. (1985) The epidemiology of anorexia nervosa and bulimia nervosa. Journal of Psychiatric Research, 19, 143-153.

Timimi, S. \& Robinson, P. (1996) Disturbances in children of patients with eating disorders. European Eating Disorder Review, 4, 183-188.

Treasure, J. L., Troop, N. A. \& Ward, A. (1996) An approach to planning services for bulimia nervosa. British Journal of Psychiatry, 169, 551-554.

Van Wezel-Meijler, G. \& Wit, J. M. (1989) The offspring of mothers with anorexia nervosa: a high risk group for under-nutrition and stunting? European Journal of Paediatrics, 149, 130-135.

Woodside, D. \& Shekter-Wolfson, L. (1990) Parenting by patients with anorexia nervosa and bulimia nervosa. International Journal of Eating Disorders, 9, 303-309.

World Health Organization (1992) The Tenth Revision of the International Classification of Diseases and Related Health Problems (ICD-10). Geneva:WHO.

\section{Multiple choice questions}

1. Regarding mothers with eating disorders:

a eating-disordered symptomatology frequently rises during the postpartum period

b eating-disordered symptomatology frequently rises during pregnancy

c eating-disordered mothers tend to misperceive the size of their infants

d children of parents with eating disorders are at increased risk of developing disturbance.

e children's eating and feeding disorders may be specifically linked with maternal eating habits and attitudes.

2. Children with 'selective eating disorder':

a initially present as having food fads

b typically eat only three or four different foods

c usually increase their range of foods as they grow older

d usually present at clinic as physically healthy, with weight and height in the normal range

b 'food avoidance emotional disorder' (FAED) in children meets the diagnostic criteria for anorexia nervosa.

3. Mothers with bulimia nervosa frequently:

a fear that feeding their child may trigger a binge

b intensely dislike their child making a mess at mealtimes

c encourage their infants to self-feed

d miss or misperceive their infant's cues at mealtimes

e get into conflict with the children over food refusal/mess.

\begin{tabular}{cll}
\hline MCQ answers & & \\
1 & 2 & 3 \\
a T & a T & a T \\
b F & b T & b T \\
c F & c F & c F \\
d T & d T & d T \\
e T & e F & e T
\end{tabular}

